\title{
Analysis of JAK-STAT signaling pathway genes and their microRNAs in the intestinal mucosa of genetically disparate chicken lines induced with necrotic enteritis
}

Anh Duc Truong ${ }^{1}$, Deivendran Rengaraj ${ }^{1}$, Yeojin $\operatorname{Hong}^{1}$, Cong Thanh Hoang ${ }^{1}$, Yeong Ho Hong ${ }^{{ }^{*}}$, and Hyun S. Lillehoj ${ }^{2}$

${ }^{1}$ Department of Animal Science and Technology, Chung-Ang University, Anseong 17546, Republic of Korea

${ }^{2}$ Animal Biosciences and Biotechnology Laboratory, Agricultural Research Services, United States Department of Agriculture, Beltsville, MD 20705, USA

*Corresponding author:

Yeong Ho Hong, Ph.D.

Department of Animal Science and Technology,

Chung-Ang University, Anseong 17546,

Republic of Korea

Tel: +82-31-670-3025, Fax: +82-31-671-3025

E-mail: yhong@cau.ac.kr 


\section{Abstract}

The JAK-STAT signaling pathway plays a key role in cytokine and growth factor activation and is involved in several cellular functions and diseases. The main objective of this study was to investigate the expression of candidate JAK-STAT pathway genes and their regulators and interactors in the intestinal mucosal layer of two genetically disparate chicken lines [Marek's disease (MD)-resistant line 6.3 and MD-susceptible line 7.2] induced with necrotic enteritis (NE). Through RNA-sequencing, we investigated 116 JAK-STAT signaling pathway-related genes that were significant and differentially expressed between the intestinal mucosa of the two lines compared with respective uninfected controls. About 15 JAK-STAT pathway genes were further verified by qRT-PCR, and the results were in agreement with our sequencing data. All the identified 116 genes were annotated through Gene Ontology and mapped to the KEGG chicken JAK-STAT signaling pathway. To the best of our knowledge, this is the first study to represent the transcriptional analysis of a large number of candidate genes, regulators, and potential interactors in the JAK-STAT pathway of the two chicken lines induced with NE. Several key genes of the interactome, namely, STAT1/3/4, STAT5B, JAK1-3, TYK2, AKT1/3, SOCS1-5, PIAS1/2/4, PTPN6/11, and PIK3, were determined to be differentially expressed in the two lines. Moreover, we detected 68 known miRNAs variably targeting JAK-STAT pathway genes and differentially expressed in the two lines induced with NE. The RNA-sequencing and bioinformatics analyses in this study provided an abundance of data that will be useful for future studies on JAK-STAT pathways associated with the functions of two genetically disparate chicken lines induced with NE.

Keywords: Chicken, necrotic enteritis, JAK-STAT signaling, miRNAs. 


\section{Introduction}

In mammals, the JAK-STAT pathway is activated by more than 40 cytokines and growth factors, and is involved in several cell functions such as differentiation, proliferation, and apoptosis. To activate the JAK-STAT pathway, the cytokines have to first interact with their corresponding receptors and activate receptor-associated members of the JAK family (JAK1, JAK2, JAK3, and TYK2). Then, JAKs activate docking sites of STAT1-6 and other signaling molecules and induce their subsequent translocation into the nucleus where they modulate the expression of target genes (Kiu and Nicholson, 2012). Several studies demonstrated that JAK1 interacts with class I cytokine receptors in humans to stimulate signals from the IL-2 receptor family (IL-2R, IL-7R, IL-9R, and IL-15R), IL-4 receptor family (IL-4R and IL-13R), and gp130 receptor family (IL-6R, IL-11R, LIF-R, OSM-R, CT-1R, CNTF-R, NNT-1R/BSF-3R, and Leptin-R) (Schindler and Strehlow, 2000 and Zgheib et al., 2013). In addition, JAK1 interacts with class II cytokine receptors to stimulate signals from IFN-R and IL-10R (Eulenfeld et al., 2012 and Yamauchi-Takihara and Kishimoto, 2000). Similarly, JAK2 stimulates signals from the single-chain receptor family (Epo-R, Tpo-R, GH-R, and PRL-R) and IL-3 receptor family (IL-3R, IL-5R, and GM-CSF-R)(Schindler and Strehlow, 2000 and Zgheib et al., 2013). JAK3 stimulates signals from the common gamma chain $(\gamma \mathrm{C})$ receptor family (IL-4R and IL-21R) (Kaminski et al., 2010 and O'Sullivan et al., 2007). TYK2 was reported to elicit signals from IFN- $\alpha$, IL-6, IL-10, and IL-12 (Schindler and Strehlow, 2000 and Shanker et al., 2013). Recently, it has been reported that members of the SOCS family, which comprise at least eight proteins, are inducible endogenous regulators of the JAK-STAT pathway (Chaves de Souza et al., 2013).

The expression and functions of JAK-STAT pathway-related genes have been well demonstrated in mammalian species. In particular, JAK-STAT pathway dysregulation results in the disruption of several signaling pathways and causes immune deficiency syndromes (Aaronson and Horvath, 2002). However, studies in relation to JAK-STAT signaling pathway genes in avian species are limited. The aim of this study was to analyze the JAK-STAT signaling pathway cascade 
that mediates the functions of the intestinal mucosal layer and the innate immune response of two genetically disparate chickens infected with bacterial and protozoan pathogens. We performed RNA sequencing (RNA-Seq) and quantitative real-time PCR (qRT-PCR) analyses to evaluate the expression of JAK-STAT pathway-related genes in the intestinal mucosal layer of two chicken lines [Marek's disease (MD)-resistant line 6.3 and MD-susceptible line 7.2] with necrotic enteritis (NE) induced and uninfected control. Moreover, we reported the potential miRNAs targeting the identified JAK-STAT signaling pathway genes in NE-induced chickens compared with respective controls.

\section{Materials and Methods}

\subsection{Experimental birds and care}

The two White Leghorn chicken lines, the MD-resistant line 6.3 and MD-susceptible line 7.2, were obtained from the Avian Disease and Oncology Laboratory (East Lansing, MI, USA) of the Agricultural Research Service of the United States Department of Agriculture (USDA). Care and experimental use of the birds were approved by the Beltsville Area Institutional Animal Care and Use Committee of the USDA-ARS. All the experiments including bird management, experimental induction of NE, and sample collection were performed at USDA-ARS, Beltsville, USA.

\subsection{Experimental disease model for $N E$}

NE was experimentally induced in lines 6.3 and 7.2 chickens as previously described (Jang et al., 2012). Briefly, chickens were infected with E. maxima $\left(1.0 \times 10^{4}\right.$ oocysts/bird) by oral gavage

on day 14 , followed by oral gavage with a field strain of $C$. perfringens strain Del-1 $\left(1.0 \times 10^{9}\right.$ colony forming units [CFU]/bird) after 4 days. For the development of NE, the birds were fed a non-medicated commercial basal ration of $17 \%$ crude protein from 1 to 18 days of age, and the feed was replaced with a commercial non-medicated feed containing $24 \%$ crude protein at $18-20$ days of age. Chickens were raised in Petersime starter brooder units, provided ad libitum access to water, and maintained under uniform standard management conditions. The experimental and control 
groups were kept in separate rooms to prevent cross-contamination throughout the course of the experiment.

\subsection{Experimental sample collection and high-throughput RNA-Seq}

Intestinal mucosa samples (ileum: Meckel's diverticulum to $5 \mathrm{~cm}$ proximal to the ileocecal junction) were collected from NE-induced and control lines of 6.3 and 7.2 chickens (five each) on post-hatch day 20, according to a previously described protocol (Hong et al., 2012). Total RNA was extracted from all samples and used for high-throughput RNA-Seq and data analyses as previously described (Truong et al., 2015).

\subsection{JAK-STAT signaling pathway enrichment analysis}

In our previous study (Truong et al., 2015), the total raw reads generated after processing of sequencing data was over 38 million reads for the MD-resistant line 6.3 and over 40 million reads for the MD-susceptible line 7.2. Alignment of the sequence reads with the Gallus gallus (chicken) genome database revealed the expression of over 29,900 transcripts affected by NE in these two lines. Of these, 7,841 genes and 2,919 genes were significantly upregulated and downregulated, respectively, in EM/CP-infected line 6.3 compared with the uninfected control. Approximately 6,043 genes and 2,764 genes were also significantly upregulated and downregulated, respectively, in EM/CP-infected line 7.2 compared with the uninfected control. The significantly upregulated and downregulated genes which are obtained from NE-induced lines 6.3 and 7.2 in our previous study were utilized in the present study. All those genes were subjected to identify their involvement in the chicken JAK-STAT pathway according to the Kyoto Encyclopedia of Genes and Genomes (KEGG: http://www.kegg.jp/) database using DAVID Bioinformatics Resources version 6.7 (http://david.abcc.ncifcrf.gov/tools.jsp).

\subsection{Hierarchical clustering, Gene Ontology $(G O)$, and interaction analyses}

The genes identified in chicken JAK-STAT pathway were subjected to hierarchical clustering using Cluster (MeV v4.9: www.tm4.org) and Java Treeview software 
(http://jtreeview.sourceforge.net/). Cluster map analysis of genes identified in the two lines was performed using Euclidean distance. The p values were calculated using the right-tailed Fisher's exact test. Next, GO functional enrichment analysis of JAK-STAT pathway genes identified in the two lines was performed using Blast2GO (version 2.7.1) (http://www.blast2go.org/). Finally, cytokines and JAK-STAT signaling pathway gene interactions were analyzed using the Search Tool for the Retrieval of Interacting Genes/Proteins (STRING) program (version 10), a database of known and predicted gene/protein interactions (Szklarczyk et al., 2015).

\subsection{Real-time PCR verification of JAK-STAT pathway genes}

Real-time PCR was used to confirm the differential expression of JAK-STAT pathway genes revealed by RNA-Seq. Primer sequences of 15 genes and the internal control gene glyceraldehyde3-phosphate dehydrogenase (GAPDH) were designed using Lasergene software (DNASTAR Inc. Madison, WI, USA) and synthesized by Genotech Co. Ltd. (Daejeon, South Korea) (Table 1). cDNA synthesis was performed using Maxima First Strand cDNA Synthesis Kit (Thermo Scientific) according to the manufacturer's recommendations. qRT-PCR was performed using $2 \times$ Power SYBR Green Master Mix (Roche, IN, USA) according to the manufacturer's instructions with a

LightCycler 96 system (Roche). Relative gene-specific expression was calculated using the $2^{-\Delta \Delta C t}$ method after normalization with chicken GAPDH (Livak and Schmittgen, 2001). All qRT-PCR analyses were performed in triplicate.

\subsection{Prediction of miRNAs targeting JAK-STAT pathway genes}

For analysis, miRNAs targeting JAK-STAT signaling pathway genes were first predicted in silico using the miRecords database (http://c1.accurascience.com/miRecords/prediction_query.php), which integrates the following miRNA target prediction tools: miRanda, MirTarget2, miTarget, NBmiRTar, RNAhybrid, and TargetScan (Xiao et al., 2009). Next, JAK-STAT pathway genetargeting miRNAs differentially expressed in the intestinal mucosal layer of the NE-induced chicken lines 6.3 and 7.2 were identified based on our previous study (Hong et al., 2014). 


\subsection{Statistical analysis}

Statistical analysis was performed using SPSS software (SPSS 23.0 for Windows; IBM,

Chicago, IL, USA). Data are expressed as mean values \pm SE. Statistical comparisons were performed using Student's t-test for two-group comparisons, and the level of statistically significant difference was set at $P<0.05$.

\section{Results}

\subsection{JAK-STAT signaling pathway-related genes in the NE-induced chicken lines 6.3 and 7.2}

Based on our previous RNA-Seq data (Truong et al., 2015) and mapping that genes to the KEGG pathway database, we found 116 genes differentially expressed between the two NEinduced chicken lines and involved in the JAK-STAT signaling pathway (Table S1). Based on $P<$ 0.01 and fold change cut-off $\geq 2$, several genes were significantly upregulated and downregulated in the intestinal mucosal layer of the two lines. In particular, 52 genes and 9 genes were significantly upregulated and downregulated, respectively, from EM/CP co-infection in the intestinal mucosal layer of line 6.3 compared to control. On the other hand, 37 genes and 4 genes were significantly upregulated and downregulated, respectively, from EM/CP co-infection in the intestinal mucosal layer of line 7.2 compared to control. Figure 1 shows the hierarchical clustering of 116 differentially expressed genes in NE lines 6.3 and 7.2 and their involvement in the JAKSTAT signaling pathway.

\subsection{GO analysis of differentially expressed genes in the NE-induced chicken lines 6.3 and 7.2}

All the upregulated and downregulated genes in the intestinal mucosal layer of the two NE lines were categorized into three major functional groups including molecular function, cellular component, and biological process according to GO. Each major group was further divided into several subcategories. The results of GO analysis in NE line 6.3 revealed that all of the upregulated and downregulated genes were categorized into two major functional groups including biological 
process and molecular function. The biological process group was divided into 23 subcategories such as regulation of immune system process, regulation of cell differentiation, immune response, immune system process, and intracellular signal transduction. The molecular function group was divided into 9 subcategories including cytokine receptor binding and activity, protein binding, signal transducer activity, and leukemia inhibitory factor receptor activity. We could not find any GO terms associated with the cellular component for upregulated and downregulated genes (Fig. 2A).

The results of GO analysis in NE line 7.2 revealed that all of its upregulated and downregulated genes were categorized into three major functional groups including biological process, cellular component, and molecular function. The biological process group was divided into 23 subcategories such as regulation of protein metabolic process, regulation of multicellular organismal process, intracellular signal transduction, response to chemical, immune system process, etc. The cellular component group was divided into 4 subcategories including extracellular region, extracellular space, phosphatidylinositol 3-kinase complex, and extracellular region part. The molecular function group was divided into 7 subcategories such as signal transducer activity, cytokine receptor binding, and protein binding. (Fig. 2B).

Overall, in the NE line 6.3, most of the upregulated and downregulated genes fell into the subcategories protein binding and response to stimulus in terms of molecular functions and biological processes, respectively. In NE line 7.2, most of the upregulated and downregulated genes fell into the subcategories protein binding, extracellular region, and response to stimulus in terms of molecular functions, cellular component, and biological processes, respectively.

\subsection{Evaluation of the role of differentially expressed genes in the JAK-STAT signaling pathway of}

\section{NE-induced chickens}

All the differentially expressed genes (116 genes) in NE-induced lines 6.3 and 7.2 were localized to the chicken JAK-STAT signaling pathway to determine their exact role. About 91 genes and 25 genes were upregulated and downregulated, respectively, in NE-induced line 6.3 
compared with the control group. About 94 genes and 22 genes were upregulated and downregulated in NE-induced line 7.2 compared with the control group. All these differentially expressed genes were critically positioned in the KEGG chicken JAK-STAT signaling pathway. Figures $3 \mathrm{~A}$ and $3 \mathrm{~B}$ show the position of 116 genes differentially expressed in the intestinal mucosal layer of the NE-induced chicken lines 6.3 and 7.2 compared with controls, respectively.

Based on the structure and function of activated JAK-STAT (Watanabe et al., 1997), we divided cytokine activation by JAK-STAT into four groups: the $\gamma$ C family of cytokines, IFN family of cytokines, gp130 family of cytokines, and single-chain family of cytokines. The interactions of each family of cytokines with JAK-STAT pathway genes were examined using the STRING program (Fig. 4). Considering that IL-2, IL-4, IL-13, CSF2, and IL-21 belong to the $\gamma \mathrm{C}$ family of cytokines, we analyzed the interactions of these cytokines with JAK-STAT signaling pathway genes and found that these interactions function to upregulate JAK1-3/STAT4-5B/SOCS1 expression (Fig. 4A). IL-19, IL-22, and IL-26 are members of the IL-10 family; IL-28B is a member of the IFN family. The interactions between the IL-10 family, IFN family, and JAK-STAT signaling function to upregulate the expressions of JAK1/2, STAT1/3, and SOCS3 (Fig. 4B). Similar upregulated expressions of SOCS3/JAK1-2/STAT1/3/TYR2 were observed in the interactions with the IL-6, LIF, and CNF genes of the gp130 cytokine family (Fig. 4C). In addition, upregulated expressions of JAK1-2/STAT1 and 5B/SOCS1-3 were observed in the interactions with the GH and PRL genes of the single-chain cytokine family (Fig. 4D).

\subsection{Real-time PCR analysis of JAK-STAT signaling pathway genes in the NE-induced chicken lines}

\section{3 and 7.2}

To further understand the role of the JAK/STAT/SOCS signaling pathway in the two genetically disparate chicken lines induced with NE, the expressions of JAKs (JAK1-3, TYK2), STATs (STAT1, 3-5B), SOCSs (SOCS1-5, 7), and SHP2 were randomly selected and quantitatively determined by qRT-PCR. As shown in Fig. 5, after EM/CP co-infection, all JAKs, STAT1, SOCS4, and SOCS7 were significantly decreased in the intestinal mucosal layer of NE line 
6.3 compared with that of the NE line 7.2. The expression of STAT4, STAT5B, SOCS2 and SHP2 mRNA were markedly decreased in NE line 7.2 compared with the control. We compared the results obtained from qRT-PCR with those generated from RNA-Seq analysis of these transcripts. Expression trends were consistent for all transcripts in both analyses, with a correlation coefficient of $\mathrm{R}^{2}=0.927$ for line 6.3 and $\mathrm{R}^{2}=0.8676$ for line 7.2 (Fig. 6 ).

\subsection{Potential miRNAs targeting JAK-STAT signaling pathway genes in the NE-induced chicken lines 6.3 and 7.2}

Initially, all the 116 genes identified in the JAK-STAT signaling pathway were analyzed in the miRecords database to identify their potential miRNAs. In the results, miRNAs targeting 51 genes were not known. The remaining 65 genes were shown to be targets of one or more miRNAs (Table S2). Subsequently, the JAK-STAT signaling pathway gene-targeting miRNAs were compared with those in our earlier study (Hong et al., 2014) to identify their differential expression in the intestinal mucosal layer of the NE-induced chicken lines 6.3 and 7.2. The analysis revealed that approximately 68 miRNAs, variably targeting JAK-STAT signaling pathway genes, were differentially expressed in the intestinal mucosal layer of the NE-induced chicken lines 6.3 and 7.2 compared with respective controls. In particular, most of the identified miRNAs were upregulated in NE line 7.2 chickens (Table S2).

\section{Discussion}

The objective of the present study was to investigate and evaluate the expression of JAKSTAT pathway genes by RNA-Seq and qRT-PCR analyses of two genetically disparate chicken lines (line 6.3 and line 7.2) induced with NE compared with respective controls. For RNA-Seq data analysis, this required integration of the available studies on the JAK-STAT pathways by listing the components, modulators, and interactions along with their functional relations to one another to build a comprehensive JAK-STAT interactome that sheds light on the relationship between associated molecules. On the other hand, GO term enrichment of JAK-STAT pathway-related 
genes was performed. To our knowledge, the expression analysis of JAK-STAT-SOCS family genes are not clear in two genetically disparate chickens of lines 6.3 and 7.2 induced with NE disease. Therefore, the findings of RNA-Seq data analysis were further validated by qRT-PCR for randomly selected JAK-STAT-SOCS family genes in the two NE lines.

In this study, the transcriptional expression levels of candidate JAK-STAT pathway genes in the intestinal mucosal layer of NE-induced chickens were examined, and several genes demonstrated significantly altered expression between the two NE lines compared with respective controls. The expression profiles of several core genes of the interactome and their key modulators such as JAK1-3, TYK2, STAT1, STAT3, STAT4, STAT5B, SOCS1, SOCS2, SOCS4, SOCS5, PTPN6, and PTPN11, were found to be significantly different at the transcriptional level, in the two NE lines. In the RNA-Seq dataset, most of the JAK and STAT family genes were significantly upregulated in the two NE lines compared with the respective controls. Among the negative regulators of this pathway, the expressions of SOCS3 and SOCS7 were significantly upregulated in NE line 7.2 and downregulated in NE line 6.3 compared with the respective controls. On the other hand, the expressions of SOCS1, SOCS2, SOCS4, and SOCS5 were markedly upregulated in the two NE lines compared with the controls; however, the expressions of these genes in NE line 6.3 were markedly higher than of those in NE line 7.2. These results indicated that the negative feedback of the SOCS family plays an important role in the intestinal mucosa response to NE disease. Moreover, JAK-STAT-SOCS and PIAS gene expressions in the two NE lines were validated by qRT-PCR analysis, and the correlation between RNA-Seq data and qRT-PCR was determined as $\mathrm{R}^{2}=0.927$ in NE line 6.3 and $\mathrm{R}^{2}=0.867$ in NE line 7.2.

The interactions between JAK2 and STAT3-4 are also activated by IFN- $\gamma$ (Kohlhuber et al., 1997). JAK2 interacts with cytosolic proteins, mediating signals to the nucleus by activation of the STAT3 transcription factor. STAT3 can be phosphorylated on tyrosine ${ }^{705}$ by JAK upon activation and on serine ${ }^{727}$ mainly by ERK and p38MAPK (Kurdi and Booz, 2007). Previous studies indicated that JAK2-STAT3 activation is necessary for normal embryogenesis, differentiation of embryonic 
stem cells, and protection against reperfusion-induced injury in neonatal and adult cardiomyocytes (Foshay et al., 2005 and Takeda et al., 1997). STAT3 plays an important role in signal transduction after interaction of the type I interferons IFN- $\alpha$ and IFN- $\beta$ with their receptors (Farrar et al., 2000). STAT4 is important in the development of Th1 cells, acting as a member of the IL-12 signaling pathway and playing a role in IFN- $\gamma$ induction (Leonard and O'Shea, 1998). IL-18 is also able to induce IFN- $\gamma$ in a STAT4-dependent manner with either IL-12 or IFN- $\alpha$ (Matikainen et al., 2001). The combined effect of IL-12, IL-18, and IFN- $\gamma$ provides a suitable condition for T cells to differentiate into Th1/17 cells (Berenson et al., 2004). IFN- $\alpha$ also activates STAT4 in human T and NK cells, resulting in the upregulation of IFN- $\gamma$ expression (Matikainen et al., 2001). Moreover, the interaction between JAK2 and STAT3-4 play important roles in mediating cytokines (IFN- $\gamma$, IL-1 $\beta$, IL-18, IL-12, and IL-17) response to various diseases in mammalian species. Particularly, the IFN family, IL-12, and IL-17 family cytokines were induced in the rat kidney in response to ischemia and reperfusion injury (Yang et al., 2008), and autoimmune diseases such as inflammatory bowel disease, psoriasis, Crohn's and Behcet's disease and rheumatoid arthritis (O'Shea and Plenge, 2012). Increased STAT3 and STAT4 have been reported in chicken lymphoid cells treated with Lactobacillus acidophilus (Brisbin et al., 2008), which activate the expression of Th1/17 cytokines including IL-2, IL-12, IFN- $\alpha$, and IFN- $\gamma$. The upregulation of JAK2, STAT3, and STAT4 in two chicken lines induced with NE indicated that the interaction between JAK2 and STAT3-4 might play an important role in $\mathrm{T}$ cell population development in the intestinal response to NE disease.

The JAK1 gene is an upstream kinase of STAT3 protein and partially responsible for its constitutive activity. The level of JAK1 expression was found to be particularly high in chicken bursa, thymus, and B and T lymphoid cell lines, which could be due to post-translational modification that activates JAK1 kinase (Bartunek et al., 1999). The interactions between JAK1, STAT1, STAT3, or STAT5B coordinate different receptor complexes. IL-2, IL-4, IL-6, IL-7, IL-9, IL-10, IL-13, IL-21, IL-22, IFN- $\alpha$, IFN- $\beta$, and IFN- $\gamma$ signals are relayed to JAK1, and they are involved in signaling by STAT1, STAT3, and STAT5B in human (Luo and Laaja, 2004). Several 
publications in mammalian species demonstrated that the increased level of signaling genes including JAK1, STAT1, STAT3, and STAT5B in pathogen infection potently induced common Th1, Th2 or Th17 cytokines, and also control early differentiation of Th1/Th2/Th17 cells (Heltemes-Harris et al., 2011; O'Shea and Plenge, 2012; Tao et al., 2010). Upregulated JAK1 level and STAT1/3/5B mRNA in NE-induced chickens may lead to increased expressions of IL-2, IL-4, IL-6, IL-7, IL-9, IL-10, IL-22, and IFN- $\alpha$ in the two chicken lines. The expressions of IL-4, IL-6, IL-9, and IL-10 were higher in NE line 7.2 than in NE line 6.3; however, the receptor levels of IL-4, IL-6, and IL-10 were higher in NE line 6.3 than in NE line 7.2. Moreover, comparing the NEinduced chickens to control groups, the expression of 8 genes (IL-2, IL-4, IL-6, IL-9, IL-10, IL-13, IL-22, and IFN- $\alpha$ ) in NE line 6.3 and 9 genes (IL-2, IL-4, IL-6, IL-7, IL-9, IL-10, IL-13, IL-22, and IFN- $\gamma$ ) in NE line 7.2 were higher than that of respective control groups. These results clearly suggest that the interactions between JAK1 and STAT1/3/5B mRNA regulated the expression of IL and IFN family members and their receptors in the intestine in response to NE disease.

TYK2 is a JAK family protein that is activated in response to multiple cytokines, including type I IFNs, IL-6, IL-10, IL-12, and IL-23. In humans, TYK2 binds to cytokine receptors via its catalytic activity framework. TYK2 is necessary for type I IFNR expression in humans (Ragimbeau et al., 2003), and the role of TYK2 as a framework protein may involve other cytokine receptors besides type I IFNR (Ragimbeau et al., 2003). The overall effect of TYK2 activity on cytokine responses is still poorly understood in chickens. Based on RNA-Seq and qRT-PCR analyses, the upregulation of the TYK2 gene in the two NE lines demonstrated the function of TYK2 in the immune response of the two NE-induced chicken lines.

The mammalian PIAS family consists of four members: PIAS1, PIAS2, PIAS3, and PIAS4 (Kohlhuber et al., 1997 and Shuai and Liu, 2003). Previous studies have revealed the antibodies specific for PIAS proteins and identified PIAS-STAT interactions in mammalian cells. After cytokine stimulation, PIAS1, PIAS2, and PIAS3 interact with STAT1, STAT3, and STAT4, respectively (Arora et al., 2003 and Liu et al., 1998). In addition, PIAS4 was shown to be 
associated with STAT1 (Liu et al., 2001). The PIAS-STAT interaction is cytokine-dependent, and PIAS proteins do not interact with STATs in unstimulated cells. In this study, we investigated 3 PIAS genes including PISA1, PIAS2, and PIAS4. The genes were upregulated in the two NE lines and were particularly high in NE line 6.3. Genetic studies on the PIAS-mediated regulation of the JAK-STAT pathway are unclear in chickens; thus, our study may provide clues on the role of PIAS proteins in regulating the JAK-STAT signaling pathway.

MicroRNAs regulate a wide range of biological processes including embryo development, cell differentiation, cell proliferation, cell metabolism, and programmed cell death by regulating one or a few mRNA targets (Shivdasani, 2006). MicroRNAs preferentially binds to the 3'UTR of the transcribed mRNA to regulate its expression and function (Rengaraj et al., 2011). In this study, JAK-STAT signaling pathway genes were predicted to be variably target by 68 miRNAs in NEinduced chickens. Comparing the expression of miRNA-target of JAK-STAT signaling pathway genes between two NE-induced chicken lines showed that the expression of 57 miRNAs were upregulated and 11 miRNA were downregulated in the intestine of the MD-susceptible line 7.2. The majority of miRNA-target of JAK-STAT signaling pathway genes was downregulated in the intestine of the MD-resistant line 6.3. This result suggests that the expression of JAK-STAT signaling pathway genes might be more regulated at the posttranscriptional level in the intestine of NE-induced genetically disparate chicken lines. Particularly, PIK3RI was predicted to be the target of up to 36 miRNAs. The PIK3RI gene plays an important role in activating Protein-Tyr kinases through its SH2 domain and signaling pathways such as the JAK-STAT and AKT/PIK3 signaling pathways (Fahmi et al., 2013 and Fukao et al., 2002). Moreover, PIK3RI is essential in the development and induction of $\mathrm{T}$ and $\mathrm{B}$ lymphocytes, macrophage cells, and mast cells during pathogenic immune responses and cytokine production (Fahmi et al., 2013 and Fukao et al., 2002).

\section{Conclusion}

The JAK-STAT signaling pathway regulates the adaptive and innate immune arms of mucosal immunity as well as epithelial repair and regeneration. Here, we report the differential expression 
of several key members of the JAK-STAT pathway and their interactors in the intestinal mucosal layer of two chicken lines induced with NE. In addition, we found other important candidates of the JAK-STAT interactome such as STAT1/3/4, STAT5B, JAK1-3, TYK2, AKT1/3, SOCS1-5, PIAS1/2/4, PTPN6/11, and PIK3 that were differentially expressed in the two NE lines examined by RNA-Seq and qRT-PCR analyses. Moreover, we detected 68 miRNAs variably targeting JAKSTAT pathway genes and differentially expressed in the two NE lines. The RNA-Seq and bioinformatics analyses in this study provided an abundance of data that will be useful for future studies on JAK-STAT pathways associated with the functions of two genetically disparate chicken lines.

\section{Acknowledgments}

This work was, partially, supported by the Next-Generation BioGreen 21 Program (No. PJ00808401) and the National Research Foundation of Korea (NRF) grant funded by the Korea government (NRF2015R1D1A1A09059345). The inbred chicken lines were obtained from ADOL (Avian Disease and

Oncology Laboratory), USDA-Agricultural Research Service, East Lansing, MI and thanks to Dr. Hans H. Cheng.

\section{Abbreviations}

Janus kinase: JAK; Signal transducer and activator of transcription: STAT; Suppressor of cytokine signaling: SOCS; Interleukin: IL; Reverse transcription: RT; Tyrosine kinase 2: TYK2; Src homology 2-containing tyrosine phosphatase 2: SH2 (PTPN); Protein Inhibitor of Activated STAT: PIAS; V-Akt Murine Thymoma Viral Oncogene Homolog: AKT; Phosphoinositide-3Kinase Regulatory Subunit: PIK3R; Interferon: IFN; Extracellular Signal-Regulated Kinase: ERK; P38 Mitogen Activated Protein Kinase: p38MAPK; Colony Stimulating Factor: CSF; Leukemia Inhibitory Factor: LIF; Growth Hormone: GH; Prolactin: PRL; NPHS1 Nephrin: CNF; Oncostatin 
M: OSM; Novel neurotrophin-1R/B cell-stimulating factor-3R: NNT-1R/BSF-3R; Erythropoietin Receptor: Epo-R; Receptor for thrombopoietin: Tpo-R.

\section{Appendix A. Supplementary data}

The following are Supplementary data to this article:

Table S1. List of 116 genes differentially expressed in the intestinal mucosa of the NEinduced chicken lines 6.3 and 7.2 and involved in the JAK-STAT signaling pathway.

Table S2. List of 68 miRNAs variably targeting JAK-STAT signaling pathway genes and differentially expressed in the intestinal mucosa of the NE-induced chicken lines 6.3 and 7.2. 


\section{References}

Aaronson, D.S., Horvath, C.M., 2002. A road map for those who don't know JAK-STAT. Science 296, 1653-1655.

Arora, T., Liu, B., He, H., Kim, J., Murphy, T.L., Murphy, K.M., Modlin, R.L., Shuai, K., 2003. PIASx is a transcriptional co-repressor of signal transducer and activator of transcription 4. The Journal of biological chemistry 278, 21327-21330.

Bartunek, P., Koritschoner, N.P., Brett, D., Zenke, M., 1999. Molecular cloning, expression and evolutionary analysis of the avian tyrosine kinase JAK1. Gene 230, 129-136.

Berenson, L.S., Farrar, J.D., Murphy, T.L., Murphy, K.M., 2004. Frontline: absence of functional STAT4 activation despite detectable tyrosine phosphorylation induced by murine IFN-alpha. European journal of immunology 34, 2365-2374.

Brisbin, J.T., Zhou, H., Gong, J., Sabour, P., Akbari, M.R., Haghighi, H.R., Yu, H., Clarke, A., Sarson, A.J., Sharif, S., 2008. Gene expression profiling of chicken lymphoid cells after treatment with Lactobacillus acidophilus cellular components. Developmental and comparative immunology 32, 563-574.

Chaves de Souza, J.A., Nogueira, A.V., Chaves de Souza, P.P., Kim, Y.J., Silva Lobo, C., Pimentel Lopes de Oliveira, G.J., Cirelli, J.A., Garlet, G.P., Rossa, C., Jr., 2013. SOCS3 expression correlates with severity of inflammation, expression of proinflammatory cytokines, and activation of STAT3 and p38 MAPK in LPS-induced inflammation in vivo. Mediators of inflammation 2013, 650812 .

Eulenfeld, R., Dittrich, A., Khouri, C., Muller, P.J., Mutze, B., Wolf, A., Schaper, F., 2012. Interleukin-6 signalling: more than Jaks and STATs. Eur J Cell Biol 91, 486-495.

Fahmi, A., Smart, N., Punn, A., Jabr, R., Marber, M., Heads, R., 2013. p42/p44-MAPK and PI3K are sufficient for IL-6 family cytokines/gp130 to signal to hypertrophy and survival in cardiomyocytes in the absence of JAK/STAT activation. Cellular signalling 25, 898-909.

Farrar, J.D., Smith, J.D., Murphy, T.L., Murphy, K.M., 2000. Recruitment of Stat4 to the human interferon-alpha/beta receptor requires activated Stat2. The Journal of biological chemistry 275, 2693-2697.

Foshay, K., Rodriguez, G., Hoel, B., Narayan, J., Gallicano, G.I., 2005. JAK2/STAT3 directs cardiomyogenesis within murine embryonic stem cells in vitro. Stem cells (Dayton, Ohio) 23, 530-543.

Fukao, T., Tanabe, M., Terauchi, Y., Ota, T., Matsuda, S., Asano, T., Kadowaki, T., Takeuchi, T., Koyasu, S., 2002. PI3K-mediated negative feedback regulation of IL-12 production in DCs. Nat Immunol 3, 875-881.

Heltemes-Harris, L.M., Willette, M.J., Vang, K.B., Farrar, M.A., 2011. The role of STAT5 in the development, function, and transformation of B and T lymphocytes. Ann N Y Acad Sci 1217, 18-31.

Hong, Y.H., Dinh, H., Lillehoj, H.S., Song, K.D., Oh, J.D., 2014. Differential regulation of microRNA transcriptome in chicken lines resistant and susceptible to necrotic enteritis disease. Poult Sci 93, 1383-1395.

Hong, Y.H., Song, W., Lee, S.H., Lillehoj, H.S., 2012. Differential gene expression profiles of beta-defensins in the crop, intestine, and spleen using a necrotic enteritis model in 2 commercial broiler chicken lines. Poult Sci 91, 1081-1088.

Jang, S.I., Lillehoj, H.S., Lee, S.H., Lee, K.W., Lillehoj, E.P., Hong, Y.H., An, D.J., Jeong, W., Chun, J.E., Bertrand, F., Dupuis, L., Deville, S., Arous, J.B., 2012. Vaccination with Clostridium perfringens recombinant proteins in combination with Montanide ISA 71 VG adjuvant increases protection against experimental necrotic enteritis in commercial broiler chickens. Vaccine 30, 5401-5406.

Kaminski, A., Welters, H.J., Kaminski, E.R., Morgan, N.G., 2010. Human and rodent pancreatic beta-cells express IL-4 receptors and IL-4 protects against beta-cell apoptosis by activation of the PI3K and JAK/STAT pathways. Biosci Rep 30, 169-175. 
Kiu, H., Nicholson, S.E., 2012. Biology and significance of the JAK/STAT signalling pathways. Growth Factors 30, 88-106.

Kohlhuber, F., Rogers, N.C., Watling, D., Feng, J., Guschin, D., Briscoe, J., Witthuhn, B.A., Kotenko, S.V., Pestka, S., Stark, G.R., Ihle, J.N., Kerr, I.M., 1997. A JAK1/JAK2 chimera can sustain alpha and gamma interferon responses. Mol Cell Biol 17, 695-706.

Kurdi, M., Booz, G.W., 2007. Can the protective actions of JAK-STAT in the heart be exploited therapeutically? Parsing the regulation of interleukin-6-type cytokine signaling. Journal of cardiovascular pharmacology 50, 126-141.

Leonard, W.J., O'Shea, J.J., 1998. Jaks and STATs: biological implications. Annual Review of Immunology 16, 293-322.

Liu, B., Gross, M., ten Hoeve, J., Shuai, K., 2001. A transcriptional corepressor of Stat1 with an essential LXXLL signature motif. Proceedings of the National Academy of Sciences of the United States of America 98, 3203-3207.

Liu, B., Liao, J., Rao, X., Kushner, S.A., Chung, C.D., Chang, D.D., Shuai, K., 1998. Inhibition of Stat1-mediated gene activation by PIAS1. Proceedings of the National Academy of Sciences of the United States of America 95, 10626-10631.

Livak, K.J., Schmittgen, T.D., 2001. Analysis of relative gene expression data using real-time quantitative PCR and the 2(-Delta Delta C(T)) Method. Methods 25, 402-408.

Luo, C., Laaja, P., 2004. Inhibitors of JAKs/STATs and the kinases: a possible new cluster of drugs. Drug discovery today $9,268-275$.

Matikainen, S., Paananen, A., Miettinen, M., Kurimoto, M., Timonen, T., Julkunen, I., Sareneva, T., 2001. IFN-alpha and IL-18 synergistically enhance IFN-gamma production in human NK cells: differential regulation of Stat 4 activation and IFN-gamma gene expression by IFNalpha and IL-12. European journal of immunology 31, 2236-2245.

O'Shea, J.J., Plenge, R., 2012. JAK and STAT signaling molecules in immunoregulation and immune-mediated disease. Immunity 36, 542-550.

O'Sullivan, L.A., Liongue, C., Lewis, R.S., Stephenson, S.E., Ward, A.C., 2007. Cytokine receptor signaling through the Jak-Stat-Socs pathway in disease. Mol Immunol 44, 2497-2506.

Ragimbeau, J., Dondi, E., Alcover, A., Eid, P., Uze, G., Pellegrini, S., 2003. The tyrosine kinase Tyk2 controls IFNAR1 cell surface expression. The EMBO journal 22, 537-547.

Rengaraj, D., Lee, B.R., Lee, S.I., Seo, H.W., Han, J.Y., 2011. Expression patterns and miRNA regulation of DNA methyltransferases in chicken primordial germ cells. PLoS One 6 (5), e19524.

Schindler, C., Strehlow, I., 2000. Cytokines and STAT signaling. Adv Pharmacol 47, 113-174.

Shanker, V., Trincucci, G., Heim, H.M., Duong, H.T., 2013. Protein phosphatase 2A impairs IFNalpha-induced antiviral activity against the hepatitis $\mathrm{C}$ virus through the inhibition of STAT1 tyrosine phosphorylation. J Viral Hepat 20, 612-621.

Shivdasani, R.A., 2006. MicroRNAs: regulators of gene expression and cell differentiation. Blood 108, 3646-3653.

Shuai, K., Liu, B., 2003. Regulation of JAK-STAT signalling in the immune system. Nature reviews.Immunology 3, 900-911.

Sohn, S.J., Barrett, K., Van Abbema, A., Chang, C., Kohli, P.B., Kanda, H., Smith, J., Lai, Y., Zhou, A., Zhang, B., Yang, W., Williams, K., Macleod, C., Hurley, C.A., Kulagowski, J.J., Lewin-Koh, N., Dengler, H.S., Johnson, A.R., Ghilardi, N., Zak, M., Liang, J., Blair, W.S., Magnuson, S., Wu, L.C., 2013. A restricted role for TYK2 catalytic activity in human cytokine responses revealed by novel TYK2-selective inhibitors. Journal of immunology (Baltimore, Md.: 1950) 191, 2205-2216.

Szklarczyk, D., Franceschini, A., Wyder, S., Forslund, K., Heller, D., Huerta-Cepas, J., Simonovic, M., Roth, A., Santos, A., Tsafou, K.P., Kuhn, M., Bork, P., Jensen, L.J., von Mering, C., 2015. STRING v10: protein-protein interaction networks, integrated over the tree of life. Nucleic Acids Res 43, D447-452. 
Tao, L., Liu, J., Li, Z., Dai, X., Li, S., 2010. Role of the JAK-STAT pathway in proliferation and differentiation of human hypertrophic scar fibroblasts induced by connective tissue growth factor. Mol Med Rep 3, 941-945.

Takeda, K., Noguchi, K., Shi, W., Tanaka, T., Matsumoto, M., Yoshida, N., Kishimoto, T., Akira, S., 1997. Targeted disruption of the mouse Stat3 gene leads to early embryonic lethality. Proceedings of the National Academy of Sciences of the United States of America 94, 3801-3804.

Truong, A.D., Hong, Y.H., Lillehoj, H.S., 2015. High-throughput sequencing reveals differing immune responses in the intestinal mucosa of two inbred lines afflicted with necrotic enteritis. Veterinary immunology and immunopathology 166, 116-124.

Watanabe, S., Itoh, T., Arai, K., 1997. Roles of JAK kinase in human GM-CSF receptor signals. Leukemia 11 Suppl 3, 76-78.

Xiao, F., Zuo, Z., Cai, G., Kang, S., Gao, X., Li, T., 2009. miRecords: an integrated resource for microRNA-target interactions. Nucleic Acids Res 37, D105-110.

Yamauchi-Takihara, K., Kishimoto, T., 2000. Cytokines and their receptors in cardiovascular diseases--role of gp130 signalling pathway in cardiac myocyte growth and maintenance. Int J Exp Pathol 81, 1-16.

Yang, N., Luo, M., Li, R., Huang, Y., Zhang, R., Wu, Q., Wang, F., Li, Y., Yu, X., 2008. Blockage of JAK/STAT signalling attenuates renal ischaemia-reperfusion injury in rat. Nephrol Dial Transplant 23, 91-100.

Zgheib, A., Pelletier-Bonnier, E., C, L.L., Jr., Annabi, B., 2013. Selective JAK/STAT3 signalling regulates transcription of colony stimulating factor-2 and -3 in Concanavalin-A-activated mesenchymal stromal cells. Cytokine 63, 187-193. 


\section{Tables}

Table 1. Primer sequences for the real-time PCR analysis of JAK-STAT signaling pathway genes.

\begin{tabular}{|c|c|c|c|c|}
\hline Gene & Primer & Nucleotide sequence (5'-3') & Length & Accession No \\
\hline \multirow{2}{*}{ GAPDH } & Forward & TGC TGC CCA GAA CAT CAT CC & \multirow{2}{*}{142} & \multirow{2}{*}{ NM_204305 } \\
\hline & Reverse & ACG GCA GGT CAG GTC AAC AA & & \\
\hline \multirow{2}{*}{ JAK1 } & Forward & GGC TCG TGC GTG TCC TAC & \multirow{2}{*}{116} & \multirow{2}{*}{ NM_204870 } \\
\hline & Reverse & GGT CGT CCG CTT ATC GTG & & \\
\hline \multirow{2}{*}{ JAK2 } & Forward & CAG ATT TCA GGC CGT CAT TT & \multirow{2}{*}{120} & \multirow{2}{*}{ NM_001030538 } \\
\hline & Reverse & ATC CAA GAG CTC CAG TTC GTA T & & \\
\hline \multirow{2}{*}{ JAK3 } & Forward & CAG CCC CAA CCA GAT GTC & \multirow{2}{*}{106} & \multirow{2}{*}{ NM_204996 } \\
\hline & Reverse & CCG CTT GAT GCC TTT GTA G & & \\
\hline \multirow{2}{*}{ TYK2 } & Forward & GCC CCA TGC AGG AGG AAT & \multirow{2}{*}{119} & \multirow{2}{*}{ XM_427671 } \\
\hline & Reverse & CTT TGC CAC AGC CAG AAT CAC & & \\
\hline \multirow{2}{*}{ SOCS1 } & Forward & CTA CTG GGG ACC GCT GAC C & \multirow{2}{*}{117} & \multirow{2}{*}{ NM_001137648 } \\
\hline & Reverse & TTA ACA CTG ATG GCA AAG AAA CAA & & \\
\hline \multirow{2}{*}{ SOCS2 } & Forward & CTG CGG AGT GTC GGA GAT & \multirow{2}{*}{100} & \multirow{2}{*}{ NM_204540 } \\
\hline & Reverse & ATT AAG CTT TCA GGG TTG TGC & & \\
\hline \multirow{2}{*}{ SOCS3 } & Forward & GAC ACC AGC CTG CGC CTC AAG A & \multirow{2}{*}{111} & \multirow{2}{*}{ NM_204600 } \\
\hline & Reverse & GCC CGT CAC CGT GCT CCA GTA GA & & \\
\hline \multirow{2}{*}{ SOCS4 } & Forward & AGC CCC TGT AAA CCA AAA Ag & \multirow{2}{*}{105} & \multirow{2}{*}{ NM_001199108 } \\
\hline & Reverse & GGG GGA TCG GAC TCT ACC & & \\
\hline \multirow{2}{*}{ SOCS5 } & Forward & CCT CCC AAT GCC CAA ATA & \multirow{2}{*}{102} & \multirow{2}{*}{ NM_001127314 } \\
\hline & Reverse & CCC GGT CAG CTC AGT CAT A & & \\
\hline & Forward & AAG TGC GGG TGG TAT TGG & & \\
\hline SOCS7 & Reverse & ATG TAA CGG GGG TCA GAA CT & 110 & XIV_004950016 \\
\hline & Forward & TTG TAA CTT CGC TAT TGG TAT TCC & 106 & 001012914 \\
\hline SIAT & Reverse & TTC CGT GAT GTG TCT TCC TTC & 100 & 1 \\
\hline & Forward & AGG GCC AGG TGT GAA CTA CT & & \\
\hline SIA13 & Reverse & CCA GCC AGA CCC AGA AAG & 98 & NIM_001030931 \\
\hline & Forward & TTC CAA TGT CAG CCA GTT ACC & & \\
\hline STAT4 & Reverse & GCA GCA GGG GGA TTG TTA & 105 & $\mathrm{AB} 2$ \\
\hline & Forward & CCC ACC CCC ATT ACA ACA & 114 & \\
\hline S1А1ОВ & Reverse & GCA GCA GCT CCT CCA CAT & 114 & NIM_204//9 \\
\hline & Forward & ATG TTG GTG GAG GGG AGA A & & \\
\hline $\mathrm{SHPZ}$ & Reverse & GGG GCT GCT TGA GTT GC & 108 & NIM_204968 \\
\hline
\end{tabular}




\section{Figure Legends}

Fig. 1. Hierarchical clustering of 116 genes differentially expressed in the NE-induced chicken lines 6.3 and 7.2 compared with respective controls and involved in the JAK-STAT signaling pathway. Genes shown in red were upregulated, and those shown in green were downregulated in the two NE-induced lines compared with the respective control. Hierarchical clustering of genes and samples were based on Spearman's rank correlation analyses.

Fig. 2. Gene Ontology. All the upregulated and downregulated genes in the intestinal mucosal layer of (A) NE-induced line 6.3 and (B) NE-induced line 7.2 were categorized into specific functional groups according to Gene Ontology using Fisher's exact test $(P<0.01)$. The $\mathrm{x}$-axis represents the number of genes. Comparing controls, upregulated genes are shown in dark blue, and downregulated genes are shown in dark red.

Fig. 3. Localization of 116 genes differentially expressed in the intestinal mucosal layer of the NE-induced chicken (A) lines 6.3 and (B) 7.2 in the JAK-STAT signaling pathway. The position of these genes was searched and marked in the KEGG Gallus gallus (chicken) JAK-STAT signaling pathway. Comparing controls, upregulated and downregulated genes were indicated in different colors.

Fig. 4. The interactions of the (A) $\gamma \mathrm{C}$ family of cytokines, (B) IFN family of cytokines, (C) gp130 family of cytokines, and (D) single-chain family of cytokines with JAK-STAT signaling pathway genes that differentially expressed in the intestinal mucosal layer of the NE-induced chicken lines 6.3/7.2 as predicted using the STRING program (score 0.9). Arrows in the interaction network indicate the direction of action.

Fig. 5. Quantitative real-time PCR analysis of JAK-STAT and SOCS pathway genes in the intestinal mucosal layer of NE-induced lines 6.3 and 7.2 compared with respective controls. Data are expressed as mean values $\pm \mathrm{SE}(n=3)$ and significant differences in gene expression levels between NE line 6.3 and NE line 7.2 are indicated as follows: $* P<0.05$, ** $P<0.01$, and *** $P<$ 0.001 .

Fig. 6. Comparison of the $\log _{2}$ (FC) of 15 selected transcripts using RNA-Seq and qRT-PCR data on the intestinal mucosal layer of two genetically disparate NE induced chicken lines. 
Key Color (log2 Treatment/Control)

$$
9.0
$$

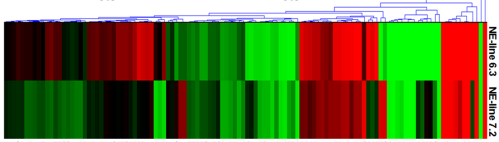

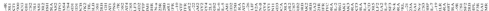
the 


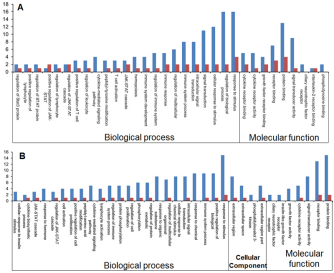




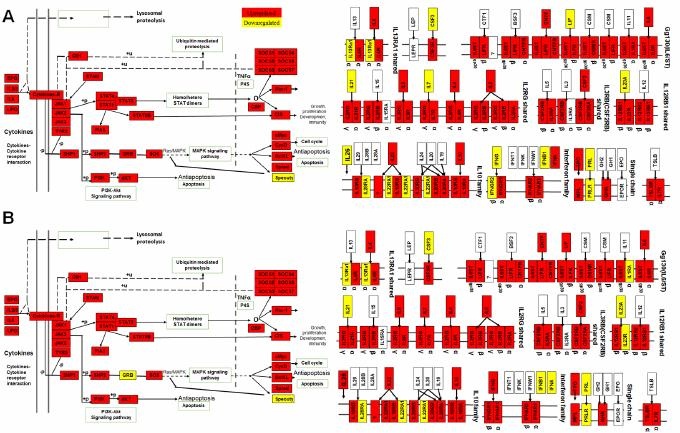




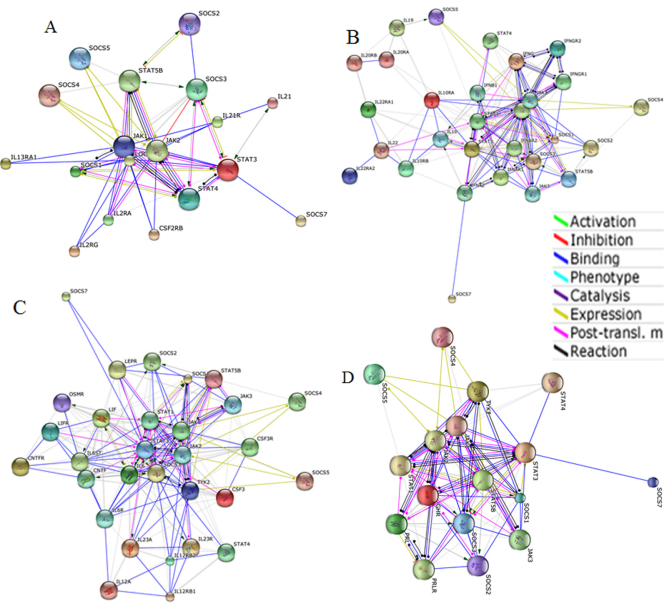




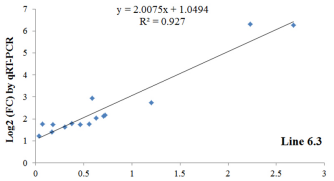

\section{Log2 (FC) by RNA-Seq}

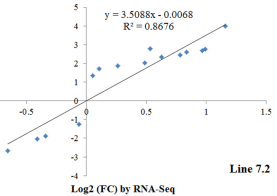

